
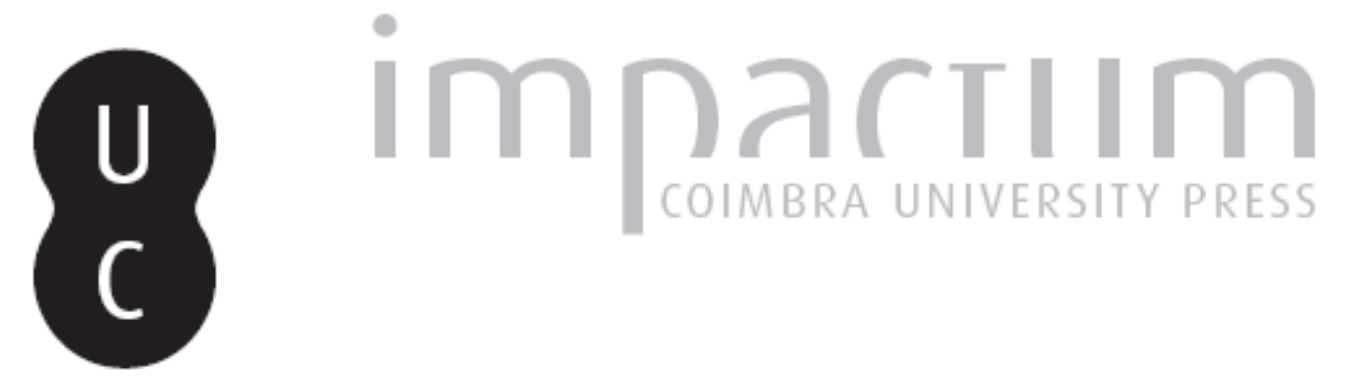

\title{
Warum gibt es keinen Mythos im Sophistes?
}

\section{Autor(es): $\quad$ Colloud-Streit, Marlis}

Publicado por: Imprensa da Universidade de Coimbra

URL persistente:

URl:http://hdl.handle.net/10316.2/42248

DOI:

DOl:https://doi.org/10.14195/2183-4105_4_2

Accessed : $\quad$ 26-Apr-2023 08:55:45

A navegação consulta e descarregamento dos títulos inseridos nas Bibliotecas Digitais UC Digitalis, UC Pombalina e UC Impactum, pressupõem a aceitação plena e sem reservas dos Termos e Condições de Uso destas Bibliotecas Digitais, disponíveis em https://digitalis.uc.pt/pt-pt/termos.

Conforme exposto nos referidos Termos e Condições de Uso, o descarregamento de títulos de acesso restrito requer uma licença válida de autorização devendo o utilizador aceder ao(s) documento(s) a partir de um endereço de IP da instituição detentora da supramencionada licença.

Ao utilizador é apenas permitido o descarregamento para uso pessoal, pelo que o emprego do(s) título(s) descarregado(s) para outro fim, designadamente comercial, carece de autorização do respetivo autor ou editor da obra.

Na medida em que todas as obras da UC Digitalis se encontram protegidas pelo Código do Direito de Autor e Direitos Conexos e demais legislação aplicável, toda a cópia, parcial ou total, deste documento, nos casos em que é legalmente admitida, deverá conter ou fazer-se acompanhar por este aviso.

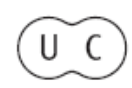




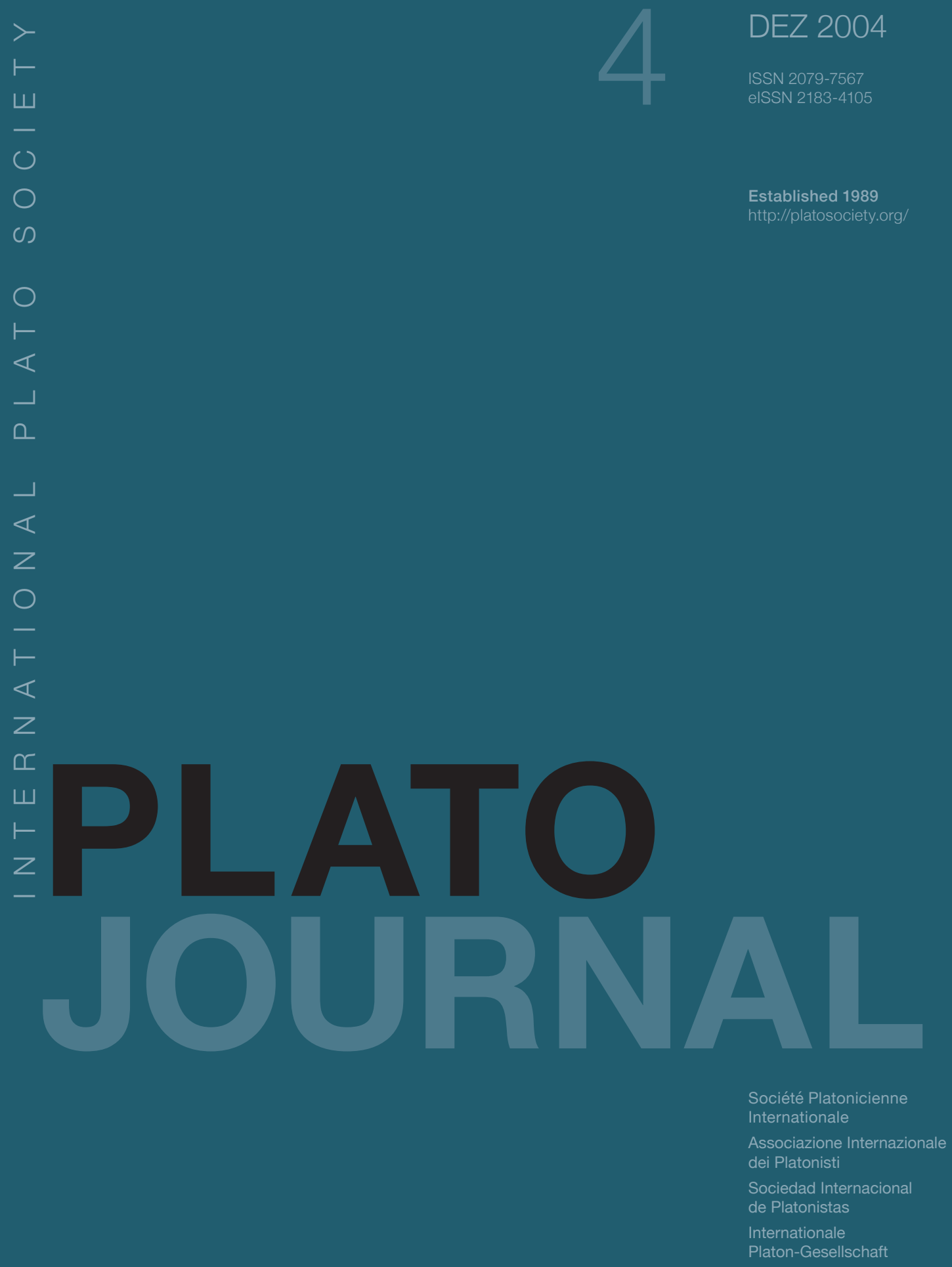




\section{WARUM GIBT ES KEINEN MYTHOS IM SOPHISTES?}

Die platonischen Dialoge Sophistes und Politikos werden gelegentlich als Zwillingsdialoge ${ }^{1}$ verstanden. Die beiden Werke seien so eng miteinander verbunden, dass sie als ein einziges Unternehmen verstanden werden sollten $^{2}$. Die enge Zusammengehörigkeit schliesst sich zum Beispiel aus der Anwesenheit derselben Protagonisten in beiden Dialogen, wenn auch teilweise mit unterschiedlicher Rollenverteilung. So ist jedesmal Theodoros, der bekannte Mathematiker, mit seinen Schülern Theaitetos und Jung-Sokrates anwesend und der Fremde aus Elea sucht jeweils mit einem von Theodoros' Schülern eine Definition. Der fremde Gast aus Elea hat ebenfalls jedes Mal die Gesprächsführung inne und Sokrates hält sich fast stumm im Hintergrund. Platon setzt das dramatische Geschehen des Sophistes und des Politikos auf den gleichen Tag an. Ausgangspunkt der Diskussion im früheren Sophistes ist das Übereinkommen zwischen den Anwesenden, nacheinander den Sophisten, den Staatsmann und den Philosophen zu bestimmen. Dieses Vorhaben wird zu Beginn des Politikos (257a1-c4) bestätigt. Kaum ist die Diskussion im Sophistes beendet, gibt sich der Fremde einverstanden, nun auch noch den Staatsmann zu bestimmen. Theaitetos wird eine Pause zugestanden. JungSokrates übernimmt die Rolle des Gesprächspartners und die Diskussion wird unmittelbar im Politikos fortgeführt. Gemeinsam ist ebenfalls der Versuch, anfangs die Definition des Sophisten respektive des Staatsmannes mittels der dihairetischen Methode zu erreichen. Sowohl im Sophistes als auch im Politikos wird in höchsten Tönen von der dialektischen Wissenschaft gesprochen. Im Sophistes wird sie als die vornehmste Wissenschaft überhaupt bezeichnet (TS Heyts, 253c $5^{3}$ ). Im Politikos erfährt der Leser, dass der Zweck der gesamten Untersuchung nicht nur eine Bestimmung des Staatsmannes sei, vielmehr gehe es darum, sich überhaupt in Dialektik zu üben (285d).

Bei aller Verbundenheit gibt es dennoch einen beachtenswerten Unterschied: Platon lässt im Sophistes den Fremden aus Elea keinen

${ }^{1}$ Zum Beispiel Wilamowitz-MoellendorfF, (1959), S. 439; Miller, (1980), S. 1.

${ }^{2}$ Zum Beispiel Notomi, (1999), S. 24.

${ }^{3}$ Die Zeilenzählung des Corpus Platonicum erfolgt nach der Ausgabe von I. BuRnet, Platonis Opera, Bde. 1-5, Oxford 1900-1907 (zahlreiche Nachdrucke). 
Mythos erzählen. Dagegen kommt im Politikos ein Mythos vor, und zwar einer von beträchtlichem Ausmass. Wie lässt sich die Abwesenheit beziehungsweise Anwesenheit eines Mythos in angeblich so eng zusammengehörigen Werken erklären? Die Frage scheint berechtigt, denn nicht nur Gemeinsamkeiten, sondern auch Unterschiede können zum Verstehen beider Werke beitragen. Die Untersuchung dieser Frage bietet meiner Ansicht nach Interpretationsmöglichkeiten, welche die beiden Werke im Hinblick auf das Handhaben der dialektischen Methode in einem neuen Licht erscheinen lassen. Der vorliegende Beitrag möchte zeigen, dass das Fehlen respektive das Vorhandensein eines Mythos mit Platons Verständnis von Dialektik zu tun hat. In einem ersten Schritt wird versucht, die Bedeutung von Dialektik im Politikos respektive im Sophistes zu beleuchten. Dann werden in einem zweiten Schritt die Figuren Theaitetos und Jung-Sokrates vorgestellt, und es wird eine Antwort auf die Frage gewagt, weshalb der Eleat nur einem von beiden einen Mythos erzählt.

Zweck dieses Artikels ist, eine Antwort vorzuschlagen, welche die Anwesenheit des Mythos im Politikos beziehungsweise dessen Abwesenheit im Sophistes erklärt. Die folgenden Überlegungen sind bei weitem nicht abgeschlossen und der Artikel ist als Anregung für eine vertiefte Forschung gedacht.

\section{DiALEKTIK IM POLITIKOS}

Die Suche nach dem Staatsmann hat als eigentliches Ziel, dialektischer zu machen ${ }^{4}$. Dialektischer werden, heisst, die Dialektik besser beherrschen. Das setzt viel Übung im Kleinen voraus ${ }^{5}$. Solche Übungen durchziehen denn auch den gesamten Politikos. Ziel des dialektischen Trainings wäre es, von allem einen Logos geben und auffassen zu können. Denn nur durch einen Logos kann das Unkörperliche als das Grösste und Schönste deutlich aufgezeigt werden ${ }^{6}$. Die Übungen, die dialektischer

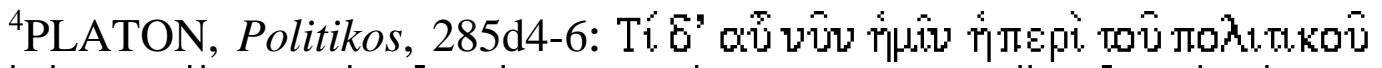

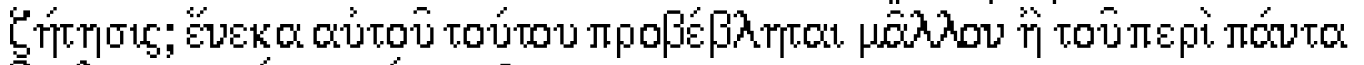
Btanektikitepois yiyeotou;

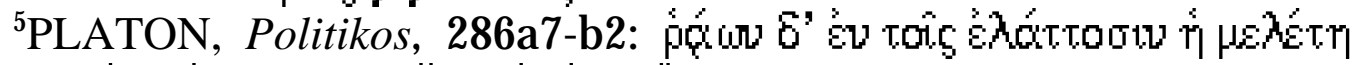

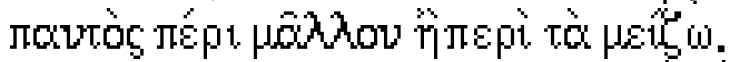

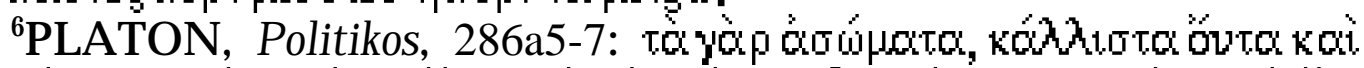

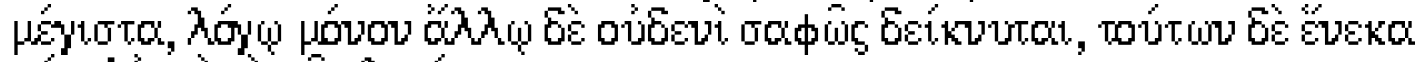

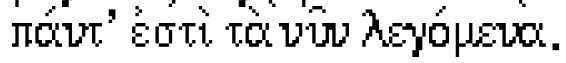


machen sollen, können in vier Etappen eingeteilt werden. Der Politikos ist ein eigentliches Training in Dialektik ${ }^{7}$. Die vier Etappen spiegeln ein buntes Bild dessen wieder, was es heisst, Dialektik zu betreiben. Das dialektische Verfahren im Politikos zeigt sich formbar und anpassungsfähig. Es begeht immer andere Wege, die zu dem

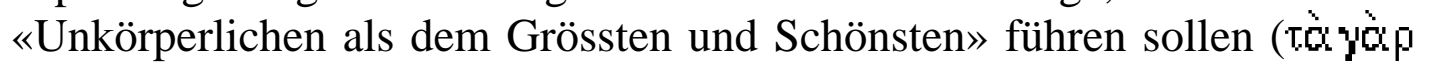
Qб sich zeigen wird, eine Übung in Dialektik. Er hilft, wie jede andere Etappe auch, dialektischer zu werden und stellt einen voll integrierten Bestandteil des dialektischen Trainings dar. Im Folgenden seien die vier Etappen des dialektischen Trainings kurz vorgestellt und gezeigt, inwiefern jede dialektischer macht. Dabei ist dem Mythos als der zweiten Etappe ein besonderes Augenmerk geschenkt.

\section{ERSTE ETAPPE}

Die erste Etappe, die zum Ziel «dialektischer werden» führen soll, heisst, die Methode der Dihairesis angemessener zu betreiben. Das meint, die Unterteilungen sollen in der Mitte und nicht irgendwo geschehen ${ }^{8}$. Eine Teilung in der Mitte entspricht am ehesten einer Trennung gemäss den natürlichen Gliedern ( $\mathrm{ka}+\mathrm{\alpha} \mu \mathrm{\lambda r}, 287 \mathrm{c3}$ ). Damit können Fehler wie eine Unterteilung der Menschen in Griechen und Barbaren vermieden werden. Die Regel, in der Mitte zu unterteilen, ist keineswegs starr. Es gibt Dinge, bei denen eine Unterteilung in zwei Hälften unangemessen ist. Ausschlaggebend ist eine Unterteilung gemäss den natürlichen Gliedern? In solchen Fällen ist eine Unterteilung gemäss den natürlichen Gliedern ausschlaggebend, wobei versucht wird, einer Zweiteilung am nächsten zu kommen.

Deutsche Zitate in Anführungs- und Schlusszeichen beruhen auf der Übersetzung von F. SCHLEIERMACHER, Platon, Werke Bde. 1-6, Darmstadt 1970, $1990^{2}$. Ansonsten handelt es sich weitgehend um meine Übersetzung.

${ }^{7}$ Ähnlich spricht DIXSAUT, (2001): «une véritable leçon de dialectique» (S. 230).

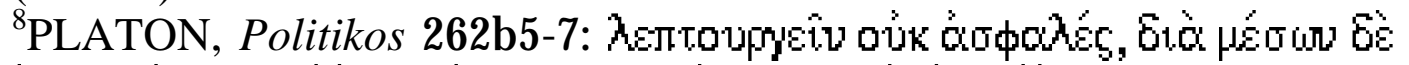

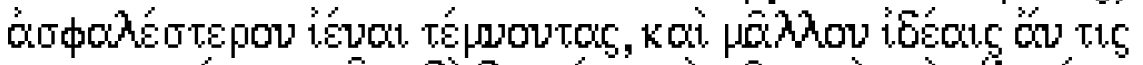

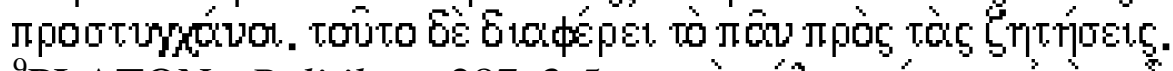
${ }^{9}$ PLATON, Politikos, 287c3-5: ka to $\mu$ hr toivun outos oilov iepeiov Btot pót

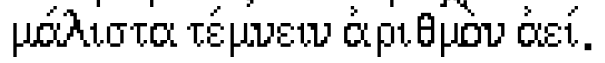




\section{ZWEITE ETAPPE}

Die zweite Etappe auf dem Pfad des dialektischen Trainings führt über den Mythos der verschiedenen Weltperioden (268e-274e). Die folgenden Zeilen versuchen $\mathrm{zu}$ zeigen, inwiefern der Mythos tatsächlich hilft, dialektischer zu werden.

Der Mythos bezieht sich direkt auf das vorausgegangene Unterteilungsverfahren. Der Fremde erwähnt zwei Fehler der Dihairesis, einen kleinen und einen grossen, die der Mythos zum Vorschein bringt. Der kleine Fehler ist, dass unpräzise gesagt wurde, auf welche Weise der Staatsmann herrscht. Der grosse Fehler besteht darin, den Hüter der Menschen als Gott anstatt als Sterblicher beschrieben zu haben. Es ist das für gleich gehalten worden, was in Wirklichkeit verschieden ist. Der Mythos ermahnt, das Andere nicht für das Selbe zu halten und bringt die Fehler in der vorausgegangenen Übung des dialektischen Trainings, der Dihairesis (258b-268d), ans Licht. Gleichzeitig macht der Mythos selbst einen Fehltritt. Dieser besteht darin, viel zu lang und viel zu pompös zu sein. Wie bisweilen Bildhauer ihre Werke grösser als nötig anlegten, so sei auch der Mythos viel zu gross geraten und habe dadurch sein eigentliches Ziel verfehlt (277a3-b6). Indem der Mythos jedoch selbst Fehler begeht, öffnet er zugleich neue Forschungswege auf der Suche nach dem Staatsmann. Eine Leistung bezüglich des dialektischen Trainings besteht also darin, Fehler aufzuzeigen und selbst Fehler zu begehen und damit weitere Forschungsmethoden anzubieten.

Der Mythos hilft weiter, dialektischer zu werden, indem er ein anderes ontologisches Niveau in die Diskussion einführt. Während bei der ersten Etappe des dialektischen Trainings vor allem körperliche Merkmale von Tieren unterschieden werden, stellt der Mythos das Körperliche dem Göttlichen gegenüber und lässt so eine ontologische Stufung erkennen. Zum Göttlichen gehört, dass es -im Gegensatz zum Körperlichen- immer dasselbe bleibt und sich immer gleich bewegt ${ }^{10}$. Die Erzählung thematisiert das Unkörperliche und Göttliche, zu dem die Dialektik strebt (269d). Der Mythos erzählt, dass das Weltall ein Lebewesen ist, das von

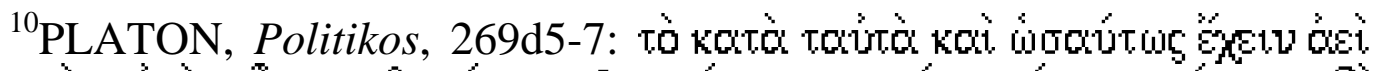

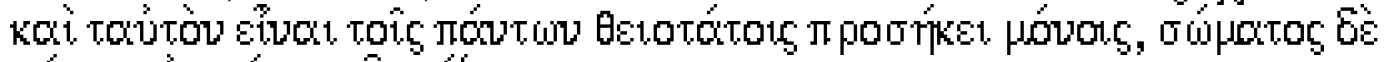

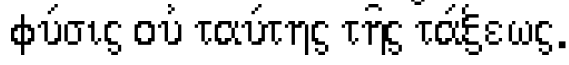




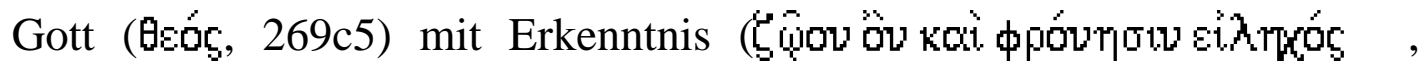
269d1) ausgestattet wurde. Diese Erkenntnis erlaubt dem Weltall, sich ohne die Hilfe des Weltenlenkers zu bewegen, wenn dieser das Ruder loslässt. Dialektisches Denken hat sein Ziel im Bereich des Intelligiblen. Die Erzählung bringt diesen Punkt in die Erörterung ein, wenn sie nahelegt, dass der Weltenlenker sich ausschliesslich mit seinem Intellekt um den gesamten Kosmos kümmert. Der mit Phronesis ausgestattete

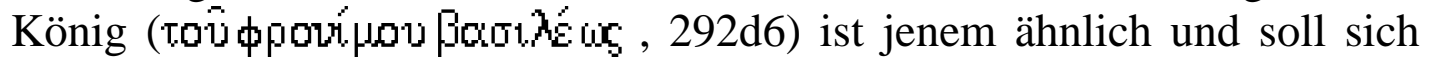
daher, in menschlichen Proportionen, durch ein Maximum an Erkenntnis auszeichnen und den göttlichen Weltenlenker nachahmen.

Der Mythos unterstützt im Weiteren das dialektische Training, weil er erfinderischer macht. Dialektischer werden, meint auch, erfinderischer (Eupetık útepov, 286e2) werden. Dinge können auf unterschiedliche Weise gesagt werden. Mit dem Mythos begibt sich der Eleat zusammen mit Jung-Sokrates auf den Weg der Auflockerung. Es soll ein wenig

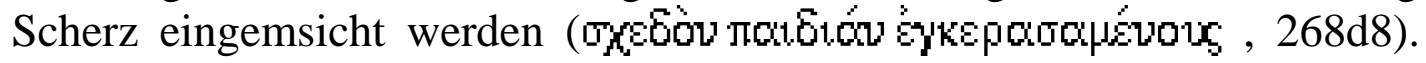
Die Entspannung erfolgt mit pädagogischen Absichten des Fremden. Der Mythos ist ein Weg, auf dem der Staatsmann gesucht wird. Es gibt andere. Erfinderischer werden, meint auch, das Gesagte nicht für bare Münze zu nehmen, sondern dem Gehörten kritisch gegenüber zu treten ${ }^{11}$. Denn wenn man es nicht zu ernst mit den Worten nimmt, ist man später reicher an Erkenntnis $^{12}$. Die Erzählung der verschiedenen Weltperioden soll einerseits den Hörer erfinderischer machen (tòvokoúoouto cupetik útepou onepjọ́rou , 286e1-2). Dieser kann beispielsweise anhand der zu lang geratenen Erzählung lernen, wie man diese auch kürzer machen könnte (i் Bpogutepo ou yevólavo, 287a2). Andererseits muss «dialektischer werden» nicht nur den Zuhörer ansprechen, sondern kann den Gesprächsleiter, also den Fremden selbst, meinen. Der Mythos bietet ihm eine Möglichkeit, das, was er sagen will, auf eine dem Gesprächspartner angemessenere Weise zu artikulieren. Er wird erfinderischer, und lernt, wie ein Gedanke einer bestimmten Person zu vermitteln ist.

\footnotetext{
${ }^{11}$ Über die Funktion und den Inhalt des Mythos wird an verschiedenen Stellen geurteilt: 268d-269c, 270b3, 273e4-6, 274e1-3, 275b1-6, 277a-c.

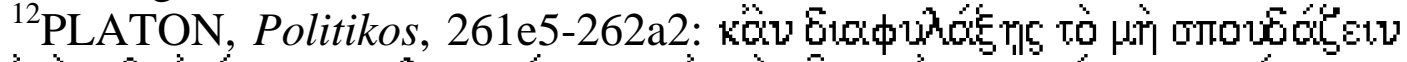

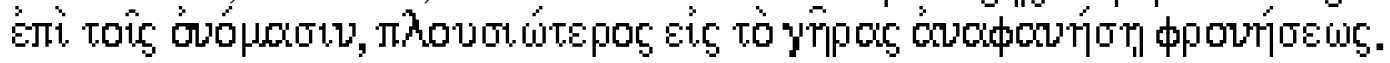


Der Mythos macht auch erfinderischer, insofern er neue Gedanken ins Werk einbringt, die wichtig bei der Suche des Staatsmannes sind und die bis anhin vernachlässigt wurden ${ }^{13}$.

\section{DRITTE ETAPPE}

Die dritte Etappe auf dem Weg dazu, dialektischer zu werden, führt über die Methode der Paradigmata. Sie helfen mit, dialektischer zu werden, indem sie von Unkenntnis zu einer wahren Meinung führen

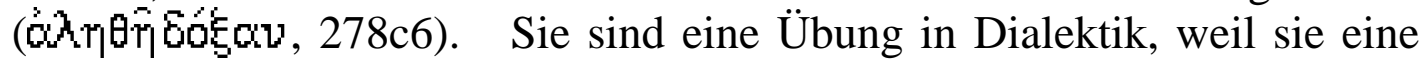
Methode trainieren, in unserem Falle, das Teilungsprozedere. Paradigmata machen dialektischer, weil sie erlauben, intellektuelle Fortschritte zu machen; denn wenn man trainiert, von einem kleinen Ding einen Logos verstehen und geben zu können, dann, um später von grösseren Dingen dazu fähig zu sein. Paradigmata braucht es, weil es schwierig ist, etwas

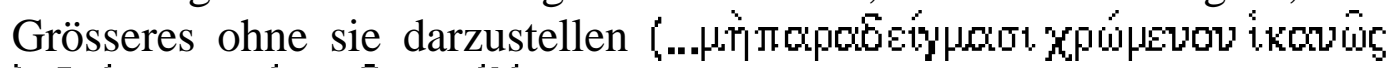

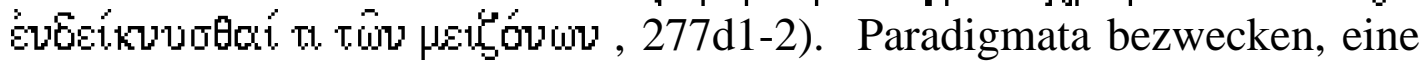
Realität grösseren Ausmasses augenscheinlicher zu machen. Besser zum Vorschein muss eine grössere Realität gebracht werden, weil sie unkörperlich ist (đoẅpoto, 286a5), also nicht mit den Sinnen wahrnehmbar. Ein Paradigma ist ein Vergleich anhand einer Gemeinsamkeit zwischen einer einfacheren und einer komplizierteren Realität. Die grössten und wertvollsten Dinge, so der Fremde, seien aber überhaupt nicht mehr durch handgreifliche Bilder aufzeigbar, sondern nur durch einen Logos (Aôu jóvov, 286a6). Das Weben sei ein kleines Paradigma, anhand dessen Ähnlichkeiten zum grossen Thema, dem Politisieren, deutlich gemacht werden soll (278e4). Der Fremde spricht von der «königlichen Zusammenflechtung» ( 306a1). Während die Methode der Dihairesis das Ziel hat, Dinge voneinander zu unterscheiden, haben die Paradigmata die Aufgabe, Dinge zu einer Einheit zusammenzurücken, indem sie Ähnlichkeiten aufzeigen. Indem der Fremde im Politikos die Wichtigkeit von Paradigmata betont, weist er gleichzeitig darauf hin, dass das Unterteilungsverfahren allein nur

${ }^{13}$ Ohne hier auf Details einzugehen, möchte ich die Vorwegnahme des Angemessenen (Tơ Hstp10v) erwähnen. So wird vom Mythos gesagt, er sei mapò kotpón , 277a6. Es ist der falsche Moment für eine solch ausgeschmückte Erzählung. Auch wird innerhalb des Mythos vom angemessenen Zeitpunkt der Umkehrung des Weltgeschehens gesprochen

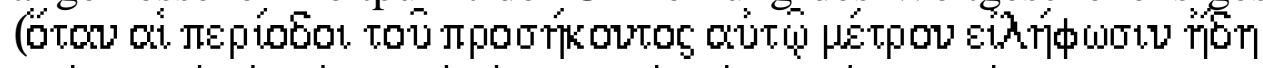

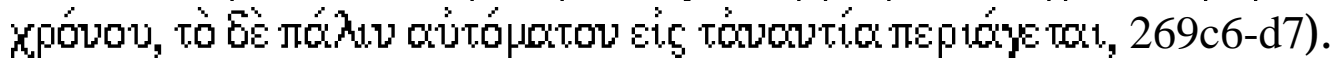


ein Teil des dialektischen Denkens bildet. Das Zusammenbringen ist ein anderer Teil.

\section{VIERTE ETAPPE}

Die vierte Etappe des dialektischen Trainings setzt sich mit den zwei verschiedenen Messarten auseinander (283b-285c). Der erste Typ zu messen, betrifft die relative Messbarkeit von quantitativen Grössen, also Grösse, Kleinheit, Länge, Dicke usw. Zum Beispiel wird das Grosse relativ zum Kleinen gemessen und umgekehrt. Den zweiten Typ nennt der

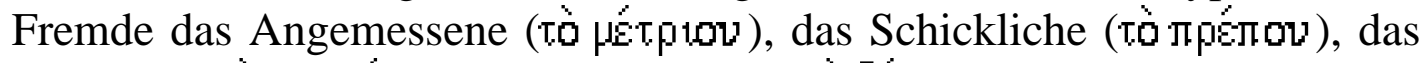
Passende (tò kat.jơv), das Gebührliche (tò bév) und «alles, was in der Mitte zwischen zwei äussersten Enden seinen Sitz hat» (лóv日' ónóod cis

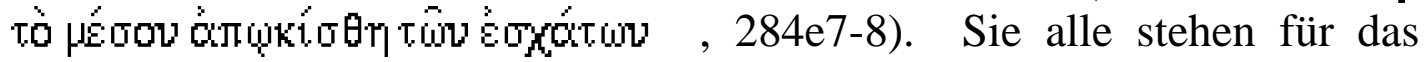
richtige Mass. So kann das Grosse auch daran gemessen werden, was angemessen ist. Die Überlegungen hinsichtlich der zwei Messarten sollen Kriterien für Übermass und Mangel liefern, und so auch eine Beurteilung erlauben, ob die vorausgegangenen langen und teils auch mühsamen Etappen des dialektischen Trainings übermässig und unnütz waren (283b). Das richtige Mass (Tó Hetplov) steht in einem engen Kontakt mit den Künsten, denn alles, was durch Künste zustande kommt, hat mit Messung zu tun ${ }^{14}$. Wer es aber nicht gewohnt ist nach Arten einzuteilen, kann zwei mögliche Fehler begehen. Der erste ist, dass zwei eigentlich verschiedene Dinge für eins gehalten werden, weil man die Dinge für ähnlich hält

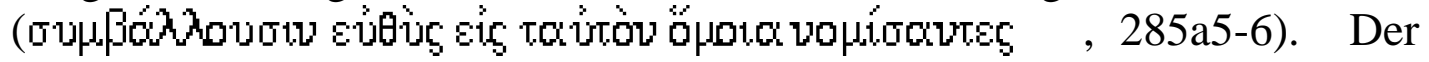
zweite mögliche Fehler besteht im Gegenteil dessen. Man hält Dinge für verschieden, die eigentlich etwas Gemeinsames haben, da man falsch

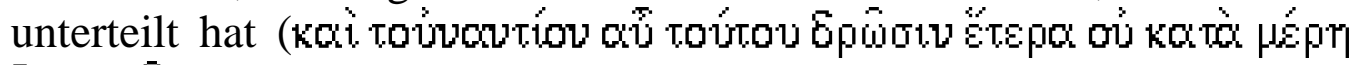
Btat.pouves, 285a6-7).

Im Anschluss an diese Überlegungen spricht der Eleat die eigentliche Absicht der Suche nach dem Staatsmann aus, die will, dass «wir in allem

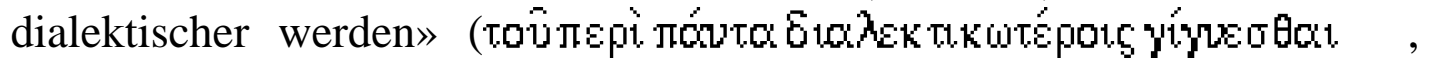
285d5-6).

Platon mag anhand der Überlegungen hinsichtlich des Angemessenen auch dafür einstehen, dass prinzipiell verschiedene Methoden zur Erforschung eines Dinges zugelassen sind, vorausgesetzt, dass diese angemessen betrieben werden.

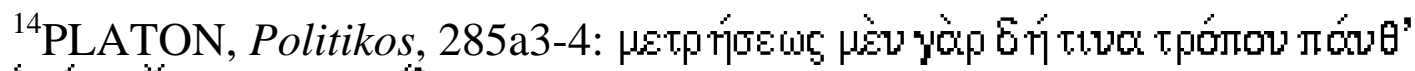

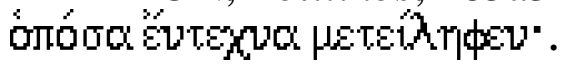


Die vier Etappen im Politikos zeigen, dass Dialektik verschiedene Methoden beinhalten kann. Alle dienen dem Ziel, dialektischer zu werden. Die folgenden Überlegungen werden zeigen, dass demgegenüber im Sophistes vor allem eine einzige Methode beleuchtet wird.

\section{DIALEKTIK IM SOPHISTES}

Der Gast aus Elea setzt im Sophistes der dialektischen Methode einen viel engeren Rahmen, als er dies im Politikos tut. Während im Politikos verschiedene Forschungsmethoden der Dialektik zur Sprache kommen, beschreibt der Eleat im Sophistes die Dialektik als die Wissenschaft, welche die Reden desjenigen führt, der richtig zeigen will,

- welche Gattungen mit welchen Gattungen übereinstimmen (תoî

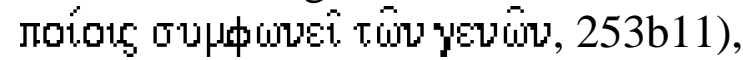

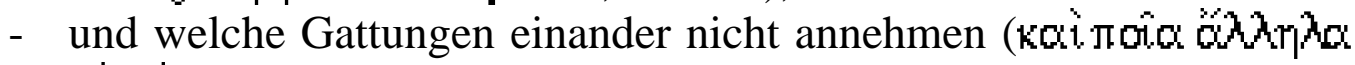
ou 6 xetot. $253 \mathrm{~b} 11-\mathrm{c} 1$ ),

- und ob es solche gibt, die vereinigend wirken und sie dadurch fähig

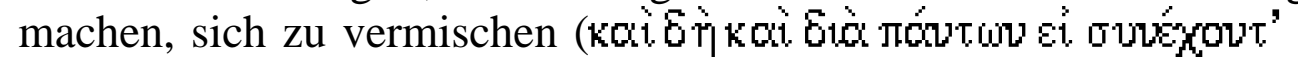

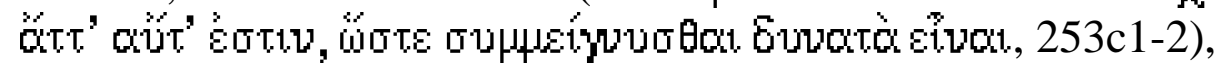

- und umgekehrt, ob es in Unterteilungen solche gibt, welche gänzlich die Ursachen der Unterteilungen sind (koi 0 atv cu tois

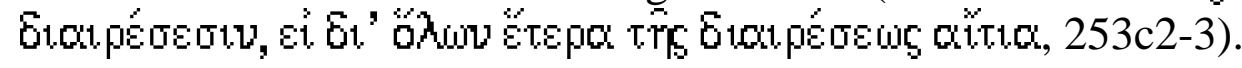

Dialektik erscheint demgemäss als Wissenschaft, die untersucht, welche Gattungen mit welchen eine Verbindung eingehen können und welche nicht, mit dem Ziel, keine bestimmte Gattung für eine andere zu halten und eine andere für diese:

«Das Trennen nach Gattungen, dass man weder dieselbe Gattung für eine andere, noch eine andere für diese halte, wollen wir nicht sagen, dies sei die dialektische Wissenschaft? ${ }^{15}$

Das Wissen darum, was miteinander in eine korrekte Verbindung treten kann und was nicht, erlaubt schliesslich einen wahren von einem falschen

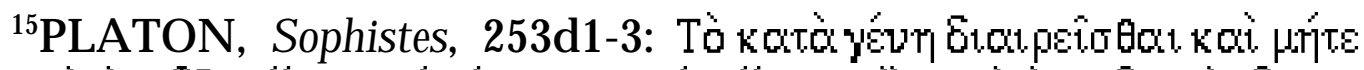

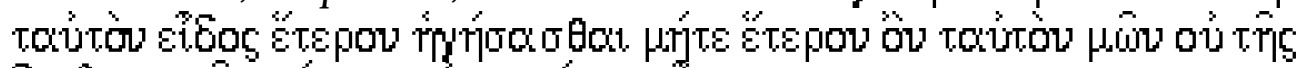

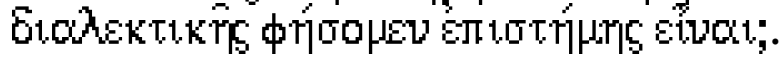


Logos $\mathrm{zu}$ unterscheiden und so den Sophisten und Philosophen auseinanderzuhalten ${ }^{16}$. Weiss man einmal um die korrekten Kombinationen von Gattungen, kann zum Unterteilungsverfahren selbst übergeleitet werden ${ }^{17}$.

Bei der Suche nach dem Sophisten, soll der Logos sichtbar machen, was denn dieser eigentlich ist ${ }^{18}$. Denn die gemeinsame Benutzung des Namens allein garantiert nicht, dass die Gesprächspartner dieselbe Vorstellung von der Sache haben (тo Epjou, to $\pi$ poy $\mu$, 218c2,4). Da die Suche nach dem Sophisten keine einfache ist, schlägt der Fremde vor, die Methode des Unterteilens zuerst am einfacheren Beispiel des Angelfischers zu üben (218c7-d2). Im Ganzen wird sieben Mal versucht, auf dem Wege des Unterteilungsverfahrens den Sophisten zu erfassen. Erst im siebten Anlauf gelangt der Fremde zu einer befriedigenden Bestimmung des Sophisten (268c-d).

Ein Leser könnte den Eindruck bekommen, so wie der Eleat im Sophistes die Wissenschaft der Dialektik beschreibt, es handle sich in erster Linie um ein Verfahren, das hauptsächlich darauf abzielt, anhand von Divisionen Gleichheit und Verschiedenheit zwischen Gattungen zu erfassen. Der Fremde lässt im Sophistes kaum erkennen, dass Dialektik auch andere Forschungsmethoden als Dihairesis beinhalten $\mathrm{kann}^{19}$. Es geht dem Eleaten offenbar darum, sich jetzt auf den Aspekt der Dialektik zu konzentrieren, der das korrekte Unterteilen zum Gegenstand hat. Das

${ }^{16}$ Dazu ausführlich NotOMI, (1999), S. 205 ff.

${ }^{17}$ Notomi, (1999), argumentiert dafür, dass die Divisionsmethode, das Wissen um die richtigen Kombinationen voraussetzt (S. 235-237); vgl. auch BLUCK, (1975), S. 127.

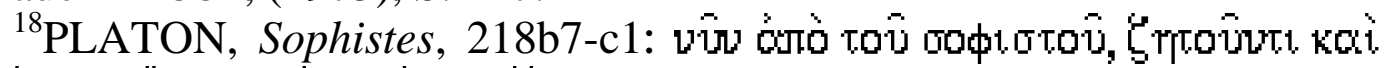

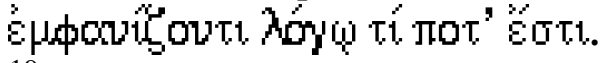

${ }^{19}$ Es gibt im Sophistes dennoch Andeutungen, ohne dass der Eleat dies explizit sagen würde, die darauf hindeuten, dass Dialektik im Sophistes mehr als Dihairesis bedeuten kann. Der Fremde benutzt erstens die zwei Paradigmata der Grammatik (253a) und der Musik (253b), um Funktionen der Dialektik aufzuzeigen. Paradigmata können Bestandteil der Dialektik sein. Zweitens sagt der Fremde, dass der Philosoph, der die Dialektik beherrscht, die Augen auf das Göttliche richtet (254a8-b1). Damit sagt der Eleat eigentlich, dass sich Dialektik mit dem Göttlichen, also mit dem Grössten und Wichtigsten, beschäftigt. Vgl. auch Notomi, (1999), S. 236; BLUCK, (1975), S. 125, die beide betonen, dass die Division ein Teil der Dialektik sei, nicht aber damit identifiziert werden sollte. 
bedeutet jedoch nicht, dass es nicht andere Verfahren innerhalb der Dialektik gibt und diese unter anderen Bedingungen herangezogen werden können. Der Fremde scheint zu denken, dass jetzt nicht der richtige Moment ist, um andere Methoden als das Unterteilungsverfahren der Dialektik heranzuziehen. Es kann geschlussfolgert werden, dass, wenn der Eleat Theaitetos keinen Mythos erzählt, das damit zusammenhängt, dass er

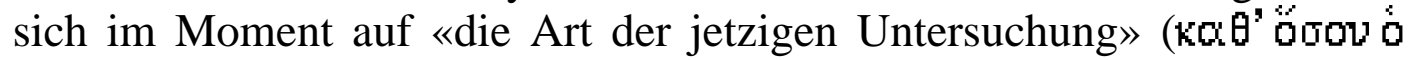

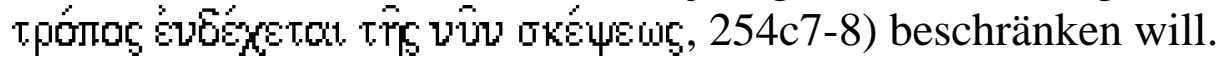

Das dialektische Verfahren im Politikos durchbricht die Starrheit des Unterteilungsverfahrens, so wie es im Sophistes beschrieben wird, genau so wie der ideale Staatsmann die Starrheit von Gesetzen durchbrechen würde (295a9-b5). Die Dialektik im Politikos begeht verschiedene Wege, die für unterschiedliche Forschungsmethoden stehen. Der Mythos bildet davon ein Weg.

Es ist in diesem Artikel noch wenig darüber gesagt worden, dass Dialektik als Kunst des Miteinanderredens auch eine Anpassung an den Gesprächspartner verlangt. Der Mythos im Politikos bringt die Anpassung des Fremden an die Persönlichkeit des jungen Sokrates besonders zum Ausdruck. Damit sei zu den Persönlichkeiten der Protagonisten von Theaitetos und Jung-Sokrates übergeleitet. Die beiden Mathematikstudenten unterscheiden sich erheblich dadurch voneinander, dass Jung-Sokrates philosophischer als Theaitetos ${ }^{20}$ ist. Philosophisch sein, meint, eine Liebe zur Weisheit in sich hegen, die einem zur Suche nach der Wahrheit auf verschiedenen Wegen vorantreibt. Jung-Sokrates hegt die Liebe zur Weisheit in einem viel stärkeren Mass als dies bei seinem Kollegen der Fall ist und er besitzt eine geistige Beweglichkeit, die Theaitetos fehlt. Dies könnten Gründe dafür sein, warum der Eleat JungSokrates einen Mythos erzählt und Theaitetos nicht.

\section{THEAITETOS}

Theodoros sagt von seinem Schüler Theaitetos, dass er nicht schön sei, sondern eine hässliche Nase und heraustretende Augen wie Sokrates habe. Trotz seiner äusseren Hässlichkeit sei Theaitetos jedoch bewunderungswürdig, denn er besitze eine ausgezeichnete Auffassungsgabe. Ausserdem zeichne sich Theaitetos durch eine

${ }^{20}$ Ich gehe davon aus, dass das Gespräch um 399 v. Chr. stattfindet. JungSokrates ist ungefähr achtzehn Jahre alt und Theaitetos ist kaum sechzehn. Vgl. dazu MiLlER, (1980), S. 8; NARCY, (1995), S. 35. 
bemerkenswerte Seelenruhe und eine Beharrlichkeit aus. Theodoros habe nicht für möglich gehalten, dass die Vereinigung von schneller Auffassungsgabe und Seelenruhe möglich sei. Denn üblicherweise gingen diese zwei Dinge nicht zusammen ${ }^{21}$. Theaitetos besitzt ausgezeichnete Kenntnisse der Geometrie und entdeckt selbst gewisse mathematische Zusammenhänge $^{22}$. Die von Platon in der Politeia geforderten mathematischen Voraussetzungen zur Dialektik besitzt er durchwegs ${ }^{23}$.

Diese Charakterisierung Theaitetos' veranlasst einige Platoninterpreten $^{24}$ zur Annahme, dass Theaitetos dem Bild der Philosophenkönige in der Politeia entspricht ${ }^{25}$. Es ist meiner Meinung nach verfehlt zu denken, dass die Beschreibung der Philosophennaturen in der Politeia auf Theaitetos zutrifft. Denn die Vereinigung von Seelenruhe mit einer raschen Auffassungsgabe zusammen mit dem Besitz ausgezeichneter Kenntnisse in Mathematik genügen nicht, um «den höchsten Wissenschaften standzuhalten» ${ }^{26}$. Es sei auf zwei Punkte eingegangen, die gewichtig genug scheinen, diese Behauptung zu rechtfertigen. Der erste Grund, weshalb Theaitetos als Philosoph nicht in Frage kommt, ist, dass er weder im Theaitetos noch im Sophistes ein starkes Begehren der Wahrheit an den Tag legt. Platon beschreibt in der Politeia die Philosophen als Menschen, die nach der gesamten Weisheit begierig sind ${ }^{27}$. Man sage nur mit Recht von jemanden, etwas zu lieben, wenn er diesem ganz zugetan $\mathrm{sei}^{28}$. Philosophen lieben immer die Kenntnisse, welche ihnen etwas offenbaren von jenem Sein, welches immer ist und nicht durch Entstehen und Vergehen unstet gemacht wird ${ }^{29}$. Kurzum die Philosophen sind diejenigen, die das Seiende selbst lieben ${ }^{30}$.

${ }^{21}$ PLATON, Theaitetos, $143 \mathrm{e}-144 \mathrm{~b}$.

${ }^{22}$ PLATON, Theaitetos, $147 \mathrm{~d}-148 \mathrm{a}$.

${ }^{23}$ PLATON, Politeia, VII 536d5-9.

${ }^{24}$ FRIEDLÄNDER, (1960), S. 135; DIÈS, (1924), S. 124; beschränkt auch ROSEN, (1983), S. 22.

${ }^{25}$ PLATON, Politeia, VI 485b-487a, VI 503c-504a, VII 535a-536c.

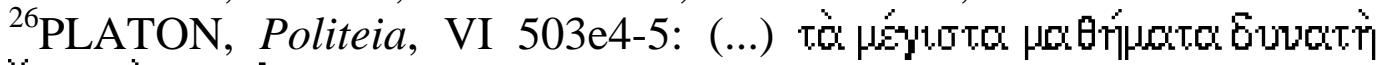
Eotou sugykeiv.

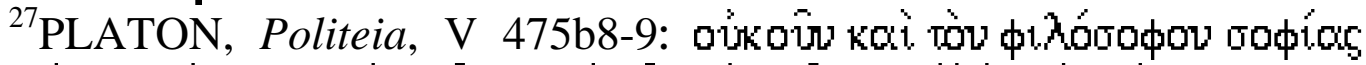

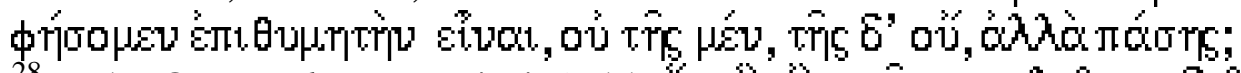

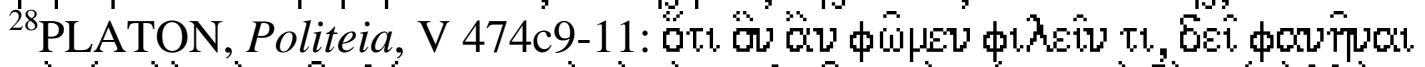

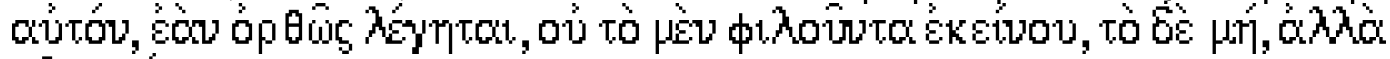

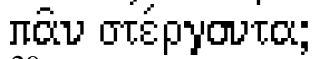

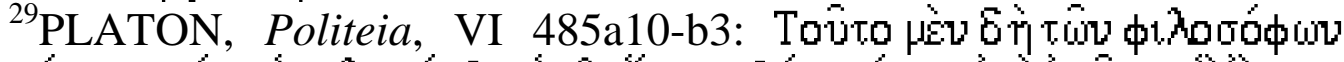

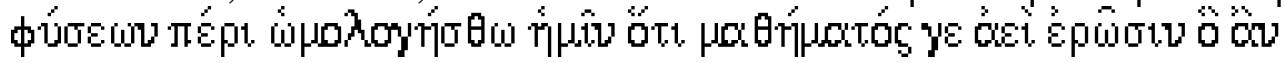


Theaitetos ist nicht so lernbegierig, gar unersättlich (on $\lambda$ †ण tus, Politeia, V 475c7) für die Weisheitssuche, wie dies Sokrates für einen Philosophen fordert. Theaitetos ist von bescheidener Natur. Der Junge lernt, wie sich Öl ausbreitet ${ }^{31}$ : Theaitetos lernt behaglich und gleichmässig mit einer andauernden Leichtigkeit. Diese Beschreibung Theodoros ist wahrscheinlich als ein Kompliment gedacht. Sie kann aber auch als eine geistige Trägheit und einen Motivationsmangel gedeutet werden. Öl fliesst einfach, leicht und behaglich. Das Bild kann auch für ein Fortschreiten ohne Begehren und ohne Anstrengung stehen. Der Philosoph dagegen begehrt das Seiende. Er wird von einer Liebe zur Weisheit getrieben. Erblickt ein Philosoph die Wahrheit, dann wird seine Seele neu befiedert. In seiner Seele beginnt es $\mathrm{zu}$ jucken und $\mathrm{zu}$ spriessen $^{32}$ und er «aufzufliegen zwar versucht, unvermögend aber und nur wie ein Vogel hinaufwärts schauend und, was drunten ist, gering achtend ${ }^{33}$. Dagegen scheint Theaitetos im Sophistes die Lust an der Untersuchung gar verloren zu haben, wenn er den Fremden fragt, «was er denn schon wieder frage»

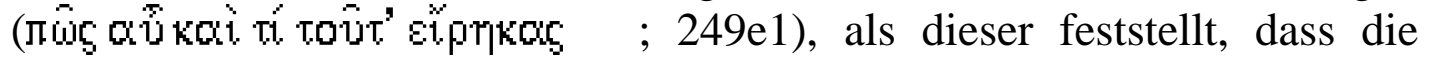
Untersuchung über das Seiende und das Nicht-Seiende ausweglos scheine (249d).

Der zweite Grund, weshalb Theaitetos als Philosoph nicht in Frage kommt, könnten seine intellektuellen Fähigkeiten sein. Er antwortet im Theaitetos mit verschiedenen Einzelbeispielen, obwohl ihm Sokrates anzeigt, dass sich Erkenntnis nicht auf Einzeldinge bezieht ${ }^{34}$. Er bestimmt im selben Werk Erkenntnis als Sinneswahrnehmung ${ }^{35}$, nachdem Sokrates

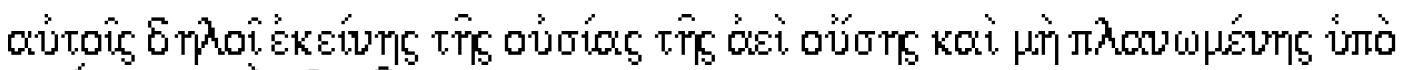
yeueoews kai qoopas.

${ }^{30}$ PLATON, Politeia, V 480a11-12: tous auto dpo Ekotov to on

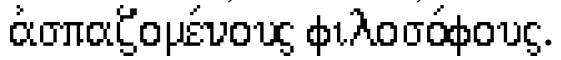

${ }^{31}$ PLATON Theaitetos, 144a-b. Man vergleiche dazu auch NARCY, (1995): «Il (Theaitetos) lui est aussi naturel d'apprendre, dit Théodore, qu' à un liquide de s'écouler: à qui apprend sans effort, est-il besoin de le désirer?» (S. 33).

${ }^{32}$ PLATON Phaidros, 251c4-5.

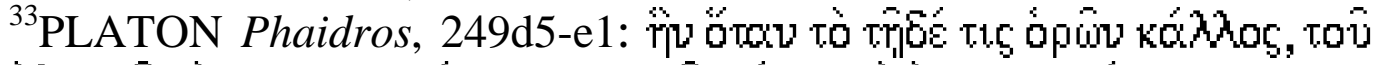
Qh

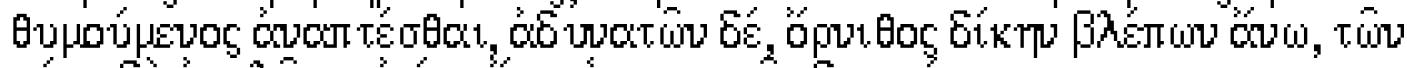

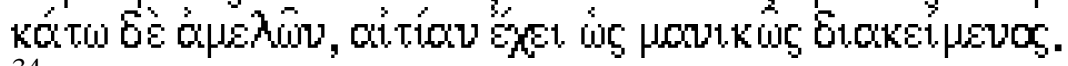

${ }^{34}$ PLATON, Theaitetos, $145 \mathrm{e} 1-3 ; 146 \mathrm{~d} 3-4$.

${ }^{35}$ PLATON, Theaitetos, 151e2-3. 
nahegelegt hat, dass Erkenntnis Wissenschaft $\mathrm{sei}^{36}$. Im Sophistes betreffen die Unterteilungen hauptsächlich Lebewesen. Theaitetos gibt sich mit den zum Teil grotesken Unterteilungen stets vollkommen einverstanden ${ }^{37}$. Theaitetos scheint auch den Faden der Diskussion verloren zu haben, wenn er darum bittet, daran erinnert zu werden, was über das All als ein Warmes

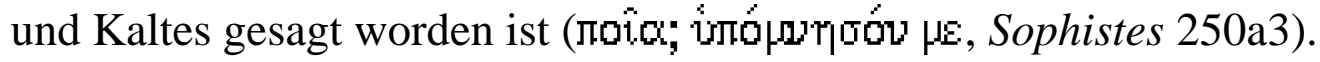

Man kann sich die Frage stellen, weshalb der Eleat mit Theaitetos im Sophistes nichts anderes, als das korrekte Unterteilen übt. Es gäbe ja auch noch andere Mittel, wie der Politikos zeigt, um zu einer Bestimmung des Sophisten zu gelangen. Theaitetos scheint zu stark im mathematischen Denken zu verharren. Wenn der Fremde im Sophistes Theaitetos keinen Mythos erzählt, dann lässt sich dies möglicherweise damit erklären, dass Theaitetos geistig zu träge ist und keinen Drang nach einem vielfältigen philosophischen Forschen verspürt. Der Junge kennt den Weg der Unterteilungen $^{38}$ und zeigt wenig Interesse daran, andere Wege kennen zu lernen. Es scheint, dass der Fremde im Sophistes denkt, es lohne sich nicht, Theaitetos auf andere Wege des Philosophierens zu führen. Der Eleat bezweckt nicht, den Jungen auf andere Wege zu führen und so vielfältig dialektischer zu machen. Deshalb beschränkt er sich auf das Unterteilungsverfahren, das Theaitetos kennt und verzichtet auf einen Mythos, der ihn möglicherweise zu grösseren Einsichten führen könnte.

\section{JUNG-SOKRATES}

Im Politikos spricht derselbe Fremde aus Elea mit Jung-Sokrates, dem Schulkollegen von Theaitetos. Jung-Sokrates scheint $\mathrm{zu}$ einem vielschichtigen Philosophieren eher geeignet $\mathrm{zu}$ sein als Theaitetos. Deshalb beschreitet der Fremde bei der Suche nach dem Staatsmann verschiedene Wege, die alle das Ziel haben, dialektischer zu machen. Ein Weg davon ist der Mythos (268e-274e), dem eine bestimmte Rolle zukommt.

Der Fremde startet die Suche nach dem Staatsmann auf dem Weg des Unterteilens. Er beginnt mit dieser Methode wahrscheinlich deshalb, weil

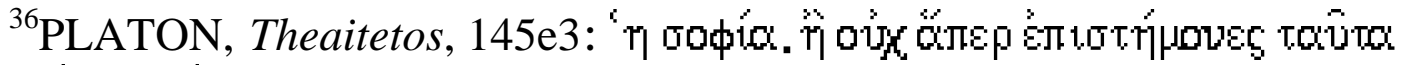
kai nopoí ;

${ }^{37}$ PLATON, Sophistes, 221c4.

${ }^{38}$ Theaitetos erzählt im gleichnamigen Werk, wie er selbst zusammen mit Jung-Sokrates versucht hat, die Zahlen einzuteilen (247e-148a).
} 
er weiss, dass Jung-Sokrates dieselben mathematischen Voraussetzungen mitbringt wie Theaitetos. Jung-Sokrates wirkt höchst motiviert. Er zeugt gar von einem gewissen Übereifer ( $\mu \curvearrowright \lambda \alpha \pi p 0 \theta 0$ ujos, 263c5), wenn er die Lebewesen in Menschen und Tiere einteilt. Enthusiasmus zeigt sich, wenn er die beiden vorgeschlagenen Wege des Fremden in der ersten Dihairesis gleichzeitig gehen will (265a7). Weil das nicht möglich ist, könnte JungSokrates sich auf einen Weg beschränken; er schlägt aber vor, beide nacheinander $\mathrm{zu}$ gehen $^{39}$. Er begeht in seiner Hastigkeit viele Unterteilungsfehler, will aber dann genau wissen, was er falsch gemacht hat $^{40}$. Die Motivation, welche Jung-Sokrates an den Tag legt, ist eigentliches Zeugnis seines Begehrens zu lernen. Der Jüngling ist in seinen geistigen Kompetenzen noch nicht ausgereift. Oft versteht er den Gedankengang des Fremden nicht auf Anhieb ${ }^{41}$. Er will aber begreifen, macht Anstrengungen und zeigt, dass er aus gemachten Fehlern lernt und sie in Zukunft zu vermeiden versucht. Er sieht zum Beispiel ein, dass sein übereifriges Vorgehen schliesslich eine Verzögerung zur Folge hat (264b4). Der Fremde schreitet mit Jung-Sokrates erst zur nächsten dialektischen Lektion, wenn er sicher ist, dass der Junge die vorausgehende Übung verstanden hat (264a8-9, 280b5-9). Es gilt, den Jungen in seinem Übereifer ein bisschen zu bremsen. Dieses Vorgehen scheint sich auszuzahlen, denn der Junge entwickelt sich im Verlaufe des Gesprächs ${ }^{42}$ und nimmt an diesem auch intensiver teil. Jung-Sokrates folgt

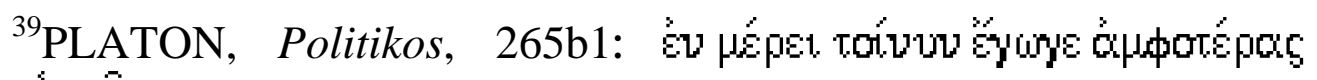
aipoUjou.

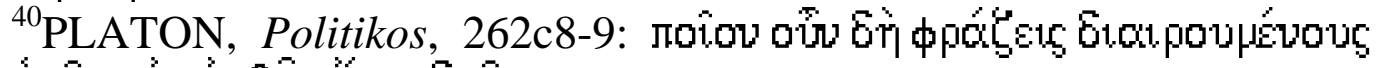
+1

${ }^{41}$ Zum Beispiel Platon, Politikos, 258a, 261c, 271c-d, 277d, 297c, 302d, $306 \mathrm{e}$.

${ }^{42}$ Jung-Sokrates lernt bereits aus den Fehlern des Unterteilungsverfahrens und sieht in den gemachten Fehlern auch einen Profit, wenn er sagt: «Sehr schön hast du mir wie die Hauptschuld die Erklärung ausgezahlt und mir noch diesen Nebenweg wie die Zinsen beigelegt, wodurch sie nun ganz

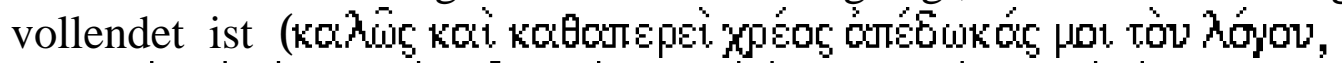

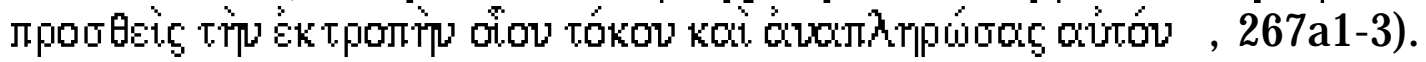
Er sieht auch die Nützlichkeit der Umwege des Unterteilens, des Mythos und der Paradigmata, die ihn lehren, weniger hastig zu sein und begangene Fehler (262a ff.) nicht zu wiederholen. Jung-Sokrates meint: «Unnützerweise scheint mir wenigstens nichts gesagt zu sein von dem,

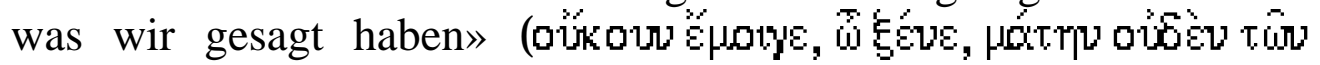

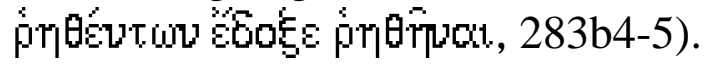


dem, was der Fremde sagt, genau. Er stellt intelligente Fragen ${ }^{43}$. Der Fremde mag denken, dass das Begehren zu lernen und die Intelligenz Jung-Sokrates erlauben, zu anderen dialektischen Lektionen, nebst der ersten Lektion der Dihairesis zu schreiten. Jung-Sokrates ist dem Fremden auch einfach sympathisch ${ }^{44}$. Das mag ebenfalls ein Grund sein, weshalb er den Jungen fördern möchte. Der Junge scheint zu einer Steigerung seiner Fähigkeiten imstande zu sein, sonst würde der Fremde kaum zu einer nächsten Lektion überleiten. Der Mythos folgt nicht ohne Grund auf die erste gescheiterte Dihairesis. Die Erzählung der verschiedenen Weltperioden reduziert das Tempo des Jungen. Damit greift Platon auf den Phaidros zurück, wo er sagt, dass Rhetorik Seelenleitung sei ${ }^{45}$. Der Mythos im Politikos ist Ausdruck einer Anpassung an den Gesprächspartner. Denn der junge Sokrates ist von der ersten Lektion in Dialektik, dem Unterteilungsverfahren (258b-267c), stark gefordert worden. Er hat eine Verschnaufpause und eine gewisse Auflockerung verdient (268d8-e1). Der Mythos ist der zweite Schritt ins dialektische Denken. Während des Mythos (268e-274e), der eine Übung in Dialektik ist, kommen Jung-Sokrates' Begehren, die Wahrheit zu finden und seine Intelligenz besonders zum Vorschein. Der Junge zeigt ein wirkliches Interesse an der Erzählung. Er stellt präzise Fragen zu deren Inhalt ${ }^{46}$. Generell spricht der Junge viel mehr während des Mythos als dies bis dahin der Fall war.

${ }^{43}$ So will er zum Beispiel wissen, wie man denn eine Gattung ( 8 ibog $)$ von einem Teil ( $\mu \xi$ pog) unterscheiden könne (263b7). Auch stellt er präzise Fragen zum Inhalt des Mythos (271a, 271c). Siehe dazu Fussnote 46.

${ }^{44}$ Das zeigt sich zum Beispiel darin, wenn er sagt: «Ich will versuchen, es dir noch deutlicher zu erklären, Sokrates, aus Wohlgefallen an deiner

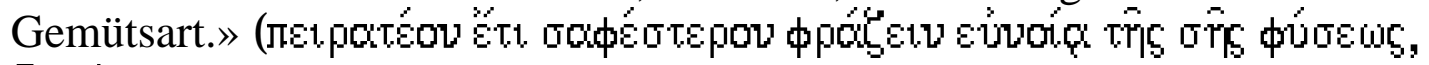

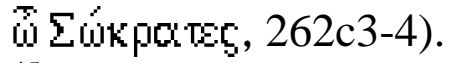

${ }^{45}$ PLATON, Phaidros, 261a7-8, 271c10.

${ }^{46}$ Er will zum Beispiel die genaue Ursache der Rückwärtsdrehung der Erde

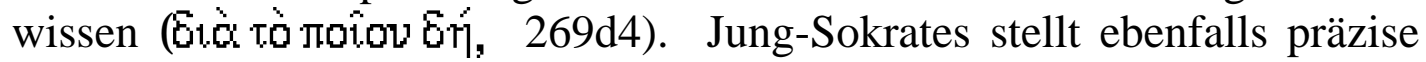
Fragen, was die Entstehungsweise in einer ehemaligen Epoche betrifft und

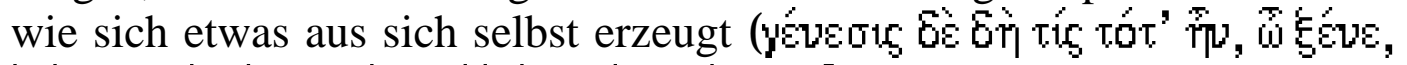

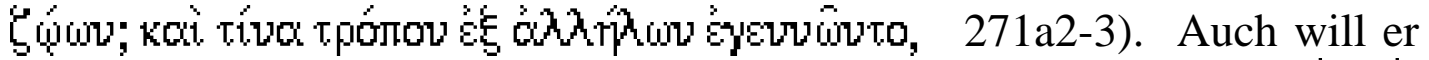
wissen, in welche Richtung sich die Welt zu Kronos Zeiten drehte ( $0 \lambda \partial$

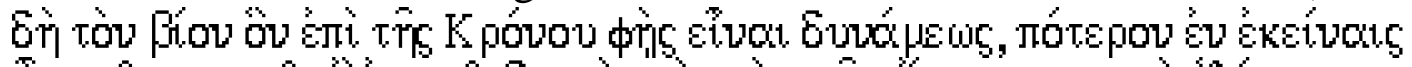

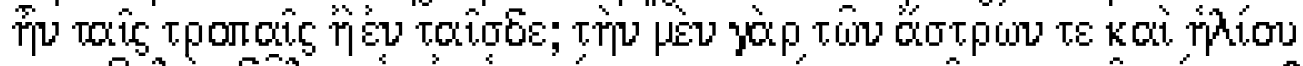

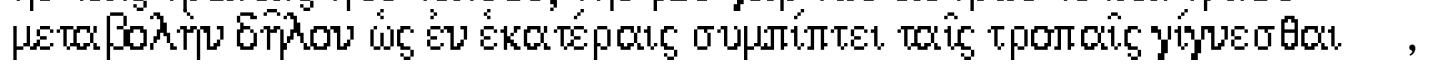
271c4-7). 
Die Umwege, die der Fremde im Politikos einschlägt, haben ihr Ziel in einer Steigerung des dialektischen Könnens. Gleichzeitig kann der Fremde anhand verschiedener Methoden testen, inwieweit es sich lohnt, mit einem Mathematikstudenten zu philosophieren. In diesem Sinne sind die verschiedenen Methoden eine Prüfung der Seele von Jung-Sokrates. Offenbar ist der Fremde der Meinung, Jung-Sokrates sei zum dialektischen Denken geeignet und es lohne sich, mit ihm zu arbeiten.

Marlis Colloud-Streit Université de Fribourg 


\section{BIBLIOGRAPHIE}

GESAMTAUSGABEN

SCHLEIERMACHER, F.: Platons Werke, Berlin 1817-1828, abgedruckt in der Sonderausgabe der Wissenschaftlichen Buchgesellschaft, hrsg. von G. Eigler, Darmstadt ${ }^{2} 1990$ (ergänzt um die Übersetzungen von Hieronymus Müller und Klaus Schöpsdau).

Platon Oeuvres complètes, Paris, Les Belles Lettres, Collection des Universités de France, Tome I-XII, 1920-1983.

SEKUNDÄRLITERATUR

BLUCK, R.: Plato's Sophist, Manchester 1975.

DiXSAUT, M.: Métamorphoses de la dialectique dans les dialogues de Platon, Paris 2001.

FrIEDLÄNDER, P.: Platon. Band III: Die platonischen Schriften: Zweite und dritte Periode, Berlin 1930, $1960^{2}$.

Miller, M.: The Philosopher in Plato's Statesman, The Hague, Boston, London 1980.

NARCY, M.: Platon Théétète, Paris 1994, $1995^{2}$.

Notomi, N.: The Unity of Plato's Sophist: Between the Sophist and the Philosopher, Cambridge 1999.

Rosen, S.: Plato's Sophist. The Drama of Original and Image, New Haven, London 1983.

Rowe, C.: Plato. Statesman, Warminster 1995.

Wilamowitz-MoellendorfF v. U.: Platon: Sein Leben und seine Werke, Berlin 1917, 19595. 